\title{
Evaluation of peripheral blood involvement of mantle cell lymphoma by fluorescence in situ hybridization in comparison with immunophenotypic and morphologic findings
}

\author{
Jun $\mathrm{Gu}^{1}$, Yang O Huh ${ }^{2}$, Feng Jiang ${ }^{1}$, Nancy P Caraway ${ }^{1}$, Jorge E Romaguera ${ }^{3}$, Tanweer M
} Zaidi $^{1}$, Ricardo L Fernandez ${ }^{1}$, Huazhong Zhang ${ }^{1}$, Issa F Khouri ${ }^{4}$ and Ruth L Katz ${ }^{1}$

${ }^{1}$ Department of Pathology; ${ }^{2}$ Department of Hematopathology; ${ }^{3}$ Department of Lymphoma and Myeloma and ${ }^{4}$ Department of Blood and Marrow Transplantation, The University of Texas MD Anderson Cancer Center, Houston, TX, USA

\begin{abstract}
Mantle cell lymphoma is non-Hodgkin's B-cell lymphoma characterized by the $t(11 ; 14)(q 13 ; q 32)$ translocation. Peripheral blood involvement of mantle cell lymphoma is usually associated with a poor prognosis and therefore, its identification is clinically important. In this study, we performed cyclin D1/lgH-probe fusion fluorescence in situ hybridization analysis on 223 peripheral blood samples: 185 from 125 mantle cell lymphoma patients, and 38 normal controls. The cutoff values for the test were established using normal controls. Flow cytometry on peripheral blood and corresponding bone marrow samples was used to evaluate this test. In all, $26 \%$ of the 185 peripheral blood samples and $27 \%$ of the 161 corresponding bone marrow samples were flow cytometry positive for mantle cell lymphoma. The mean numbers of single and- doublefusion signals and the mean number of CD5/CD19-positive cells, absolute blood lymphocyte count, and white blood cell count were significantly higher in peripheral blood and corresponding bone marrow samples with mantle cell lymphoma-positive flow cytometry. Double-fusion signals were more specific than single-fusion ones. Fluorescence in situ hybridization was far more likely to be positive for mantle cell lymphoma when the peripheral blood and the corresponding bone marrow samples had positive flow cytometry results or morphology $(P<0.01)$. Our study indicates that cyclin $\mathrm{D} 1 / \mathrm{lgH}$-fusion fluorescence in situ hybridization analysis could be used to determine the presence and character of circulating mantle cell lymphoma cells in peripheral blood, thus enhancing our ability to evaluate leukemic mantle cell lymphoma and minimum residual disease. Modern Pathology (2004) 17, 553-560, advance online publication, 5 March 2004; doi:10.1038/modpathol.3800068
\end{abstract}

Keywords: mantle cell lymphoma; interphase fluorescence in situ hybridization; peripheral blood; flow cytometry; immunophenotyping; cytomorphology

Mantle cell lymphoma is a male-predominant nonHodgkin's B-cell lymphoma. Most patients with mantle cell lymphoma have primary nodal disease with lymph node, spleen, and bone marrow involvement. Mantle cell lymphoma characteristically expresses pan-B-cell markers, monotypic surface immunoglobulin (Ig)(IgD and/or IgM), CD5, CD19, and CD $43^{1-5}$ but are typically negative for CD23 and CD10. ${ }^{6,7}$ Mantle cell lymphoma typically has the balanced chromosomal translocation

Correspondence: Dr RL Katz, MD, Department of Pathology, Unit 53, The University of Texas MD Anderson Cancer Center, 1515 Holcombe Blvd, Houston, TX 77030, USA.

E-mail: rkatz@mail.mdanderson.org

Received 20 June 2003; revised 24 September 2003; accepted 3 October 2003; published online 5 March 2004 $\mathrm{t}(11 ; 14)(\mathrm{q} 13 ; \mathrm{q} 32)$, which juxtaposes the cyclin $D 1$ gene (11q13) with the IgH gene (14q32), resulting in two nuclear fusion signals detectable by fluorescence in situ hybridization (FISH), and cyclin D1 protein overexpression detectable by immunohistochemistry. ${ }^{8-11}$

Peripheral blood involvement of mantle cell lymphoma at diagnosis has been reported in 20 $60 \%$ of patients and has been associated with a worse outcome when compared with nonleukemic mantle cell lymphoma. ${ }^{12-15}$ Lymphocytosis may be the first sign of this involvement, but it may not occur in some patients. ${ }^{12}$ As complete remission is achieved in less than $35 \%$ of mantle cell lymphoma cases, ${ }^{3,16-18}$ most patients will eventually develop relapse. The detection of overt leukemic mantle cell lymphoma or minimal residual mantle cell lympho- 
ma after the patient receives chemotherapy or other treatments requires a reliable method of monitoring circulating malignant cells in peripheral blood. The main purpose of our study was to evaluate the possibility of using FISH to determine the number of circulating cyclin D1/IgH fusion-positive cells as an index of mantle cell lymphoma involvement in peripheral blood and comparing the technique with routinely performed immunophenotypic and cytomorphologic analyses.

Recently, there have been a few studies evaluating the usefulness of the commercial cyclin D1/IgH probe set in the detection of lymph node and bone marrow involvement of mantle cell lymphoma. ${ }^{19-21}$ The sensitivity and specificity of the assay using this probe set were higher than those of classical cytogenetics, polymerase chain reaction, and immunophenotyping. However, there are only a few studies in the literature that characterized mantle cell lymphoma involvement in peripheral blood by this FISH method. Performing FISH on peripheral blood may be useful for determining precisely and rapidly whether mantle cell lymphoma has involved the peripheral blood. In order to investigate this possibility, we performed interphase FISH to detect the $t(11 ; 14)(q 13 ; q 32)$ translocation on 223 peripheral blood samples: 185 obtained from 125 mantle cell lymphoma patients and 38 from 38 apparently normal individuals for controls. Flow cytometry evidence of mantle cell lymphoma in the peripheral blood and the corresponding bone marrow samples were used as a gold standard to evaluate the FISH results.

\section{Methods}

\section{Study Group}

We collected 185 peripheral blood samples from these 125 patients with histological confirmed mantle cell lymphoma diagnosed from October 2000 to April 2002 at The University of Texas MD Anderson Cancer Center. The diagnoses were made based on morphologic and immunophenotypic findings. We had repeat samples from 41 patients with mantle cell lymphoma to compare prechemotherapy and postchemotherapy FISH results.

Peripheral blood samples were also collected from 38 apparently normal blood donors at the Gulf Coast Regional Blood Center in Houston, Texas and used as controls to establish cutoff values for the test.

In all of the mantle cell lymphoma patients, the absolute blood lymphocyte and white blood cell counts on the peripheral blood samples were tabulated. Morphologic and flow cytometry results were recorded for the peripheral blood and the corresponding bone marrow samples to evaluate the correlation between peripheral blood and bone marrow involvement. The study was conducted under the guidelines of the MD Anderson institutional review board.

\section{Sample Preparation for FISH}

The peripheral blood samples were collected in sodium-heparinized green-top vacutainers. At least $16 \mathrm{ml}$ of blood was collected from each individual. Interphase nuclei were prepared using the following hypotonic method. The samples were centrifuged at $1500 \mathrm{rpm}$ for $5 \mathrm{~min}$ at room temperature. Next, the serum was decanted from the samples, and $0.05 \mathrm{M}$ potassium chloride (hypotonic solution) was added to the tubes. The samples were then incubated at $37^{\circ} \mathrm{C}$ for $30 \mathrm{~min}$. Fresh FISH fixative solution (consisting of methanol:acetic acid, 3:1 volume/ volume) was prepared right before the incubation ended; the samples were fixed using the FISH fixative solution and washed until the cell pellets turned white. Two dropped and air-dried slides were made for each sample. One slide was stained with Diff-Quik (Harleco, Gibbstown, NJ, USA) (solution 1: methanol for $10 \mathrm{~s}$; solution 2: xanthene dye for $3 \mathrm{~s}$; solution 3: thiazine dye for $3 \mathrm{~s}$ ) and then rinsed in running tap water. The other slide was prepared for FISH.

\section{FISH Probe Set and the Cyclin D1/IgH Fusion}

The FISH studies were performed using a dual-colored, directly labeled cyclin D1 and $\operatorname{IgH}$ probe set, purchased from Vysis Inc. (Downers Grove, IL, USA), to detect fusion of the cycin D1 and IgH genes in mantle cell lymphoma cells. The IgH probe set spanned the common breakpoint regions within IgH. The cyclin D1 probe, which spanned the common break-point region centromeric to cyclin D1, had a hybridization length of approximately $370 \mathrm{~kb}$. The hybridization target of this probe extended from a point telomeric to fibroblast growth factor (FGF)4 through cyclin D1 and ended proximally at a locus about $237 \mathrm{~kb}$ centromeric to the $5^{\prime}$ end of the cyclin D1 gene.

The slides were pretreated with $2 \times$ standard saline citrate (SSC, consisting of $300 \mathrm{mM}$ sodium chloride and $30 \mathrm{mM}$ sodium citrate) for $30 \mathrm{~min}$ in a $37^{\circ} \mathrm{C}$ water bath. The slides were then dehydrated in an ethanol series $(70,85,95$, and $100 \%)$ and air-dried. A $10-\mu \mathrm{l}$ probe mixture (locus-specific identifier buffer: cyclin $\mathrm{D} 1 / \mathrm{IgH}$ probe: $\left.\mathrm{H}_{2} \mathrm{O}, 7: 1: 2\right)$ was added to each slide, and the slides were coverslipped. The slides were then sealed with rubber cement and codenatured using a Vysis HyBrite denaturation/hybridization set (Vysis Inc.) at a melting temperature of $75^{\circ} \mathrm{C}$ for $1 \mathrm{~min}$ and then hybridized at $37^{\circ} \mathrm{C}$ overnight. The slides were washed for $1 \mathrm{~min}$ in $0.4 \times \mathrm{SSC} / 0.3 \% \mathrm{NP}-40$ (Nonidet P40) at $74^{\circ} \mathrm{C}$ and transferred to $2 \times$ SSC/ $0.1 \% \mathrm{NP}-40$ at room temperature for $2 \mathrm{~min}$. Additionally, the nuclei were counterstained with $10 \mu \mathrm{l}$ of $4^{\prime}, 6^{\prime}$-diamidino-2-phenylindole dihydrochloride (DAPI) and coverslipped. 


\section{Visualization and Scoring of FISH Signals}

The IgH and cyclin D1 probes were directly labeled with Spectrum Green (Vysis, Downers Grove, IL, USA) and Spectrum Orange (Vysis, Downers Grove, IL, USA), respectively. The cells were viewed using a Leica fluorescent microscope (Bannochburn, IL, USA) equipped with a triple-band pass, DAPI/ Orange/Green (Vysis), and dual-band pass filters. The FISH samples were scored blindly in random order by two microscopists. Only nonoverlapping cells were counted. In all, 200 consecutive qualifying interphase nuclei from different areas of the same slide were scored. The slides were analyzed only if at least $80 \%$ of the cells in the field of view were interpretable and if the signals for cyclin D1, $\operatorname{IgH}$, and the fusion of the two were bright enough to be seen under the triple-band pass filter. Each nucleus was scored individually for its number of cyclin D1 (orange), IgH (green), and fusion signals. Nuclei with more than eight single-color signals were excluded. To avoid misinterpretation due to insufficient hybridization, the nuclei were counted only if at least one bright cyclin D1 (orange) and one bright $\operatorname{IgH}$ (green) signal were present. Split signals were counted as one signal if the space between them was less than the diameter of a single signal. The orange and green signals had to be either overlapping or touching (gap, $0.5 \mu \mathrm{m}$ ) to be considered a fusion. A single fusion was defined as one fusion signal per nucleus; a double fusion was defined as two fusion signals per nucleus. Possible single-fusion patterns were 101G1F (one orange, one green, and one fusion), 2O1G1F, 1O2G1F, and 2O2G1F. Possible double-fusion patterns were 1O1G2F, 2O1G2F, 1O2G2F, and 2O2G2F. A normal lymphocyte sample was used as a negative control, and a t(11;14)(q13;q32)-positive cell line was used as the control for the performance of the fusion probes. The single-fusion and double-fusion percentages were scored separately.

\section{Flow Cytometry}

In all cases, immunophenotyping was performed using dual-color or three-color flow cytometry analysis. The antibody panel included CD19, CD20, CD23, CD22, CD110, FMC7, CD10, CD5, Kappa, Lamda, IgM and IgD. All of the monoclonal antibodies were purchased from BD Biosciences Immunocytometry Systems (San Jose, CA, USA) except for FMC-7 (Immunotech, Westbrook, ME, USA), CD10 (Beckman Coulter, Miami, FL, USA), and IgM and IgD (Kallestad Laboratories, Austin, TX, USA). Flow cytometry positivity for mantle cell lymphoma was determined by looking at a panel of surface antigen markers, including the presence of monoclonality for $\kappa$ or $\lambda$ light-chain expressions, positive pan B-cell marker, and CD5/CD19 coexpression.

\section{Statistical Analysis}

Flow cytometry evidence of mantle cell lymphoma involvement in the peripheral blood and the corresponding bone marrow samples was used as a gold standard to evaluate the number of CD5/CD19positive and fusion-positive cells and the absolute blood lymphocyte and white blood cell counts in the peripheral blood samples. The number of double fusions, single fusions, CD5/CD19-positive cells, and the absolute blood lymphocyte and white blood cell counts in the peripheral blood samples were analyzed as continuous variables and were grouped as positive or negative by flow cytometry analysis. Student's $t$-test was used to compare the differences between the groups. Also, the numbers of fusionpositive cells in the peripheral blood samples from the nonpaired prechemotherapy and postchemotherapy mantle cell lymphoma groups were compared. The peripheral blood samples were divided into fusion-positive and -negative groups using a cutoff established from the 38 normal samples. The mean normal percentages of double-fusion- and singlefusion-positive cells plus three standard deviations were chosen as the cutoffs. Additionally, the $\chi^{2}$ test was used to evaluate the association between fusion FISH positivity and CD5/CD19 positivity determined by flow cytometry analysis in the peripheral blood and the corresponding bone marrow samples. The numbers of CD5/CD19-positive cells and the absolute blood lymphocyte and white blood cell counts in the fusion-positive and -negative groups were compared. The analysis was performed using the Stata 6 software program (Stata Corporation, College Station, TX, USA). In all of the statistical analyses, two-sided $P$ values $\leq 0.05$ were considered statistically significant.

\section{Results}

The cutoff values for single- and double-fusion positivity were established from a series of 38 normal blood samples. The mean percentages of single- or double-fusion-positive cells (with one standard deviation) were $6.4 \%(2.6 \%)$ and $0.2 \%$ $(0.4 \%)$, respectively. The mean percentages of single- and double-fusion-positive cells plus three standard deviations were used as cutoffs: $14.0 \%$ for single-fusion-positive samples and $1.5 \%$ for doublefusion-positive samples. Single-fusion- and doublefusion-positive mantle cell lymphoma cells are shown in Figure 1.

Using flow cytometry as a gold standard, the percentage of cells positive for double- and singlefusion signals, CD5/CD19-positive cells, and the absolute blood lymphocyte and white blood cell counts in the peripheral blood samples were compared (Figure 2). In all, 26\% of the peripheral blood samples and $27 \%$ of the corresponding bone marrow samples were flow cytometry positive for mantle cell lymphoma. When compared with flow cytometry- 


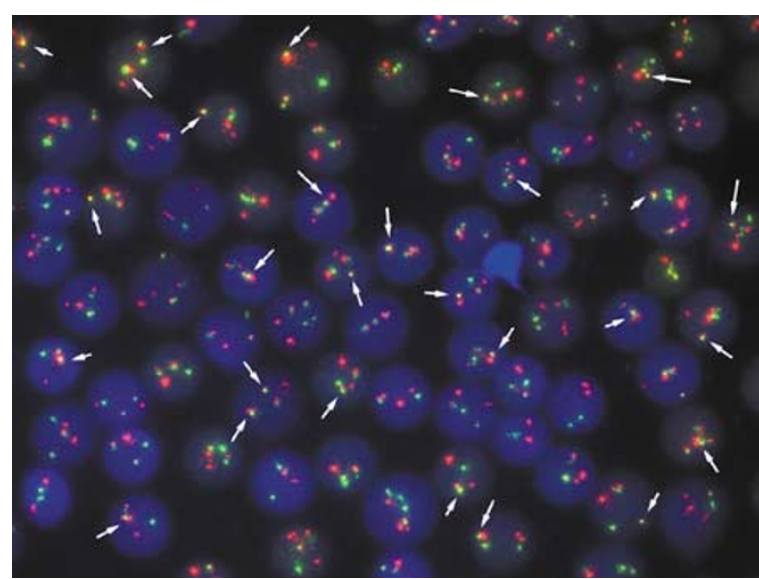

Figure 1 Cyclin D1/IgH single- and double-fusion-positive cells in peripheral blood sample showing mantle cell lymphoma involvement (FISH, × 630).
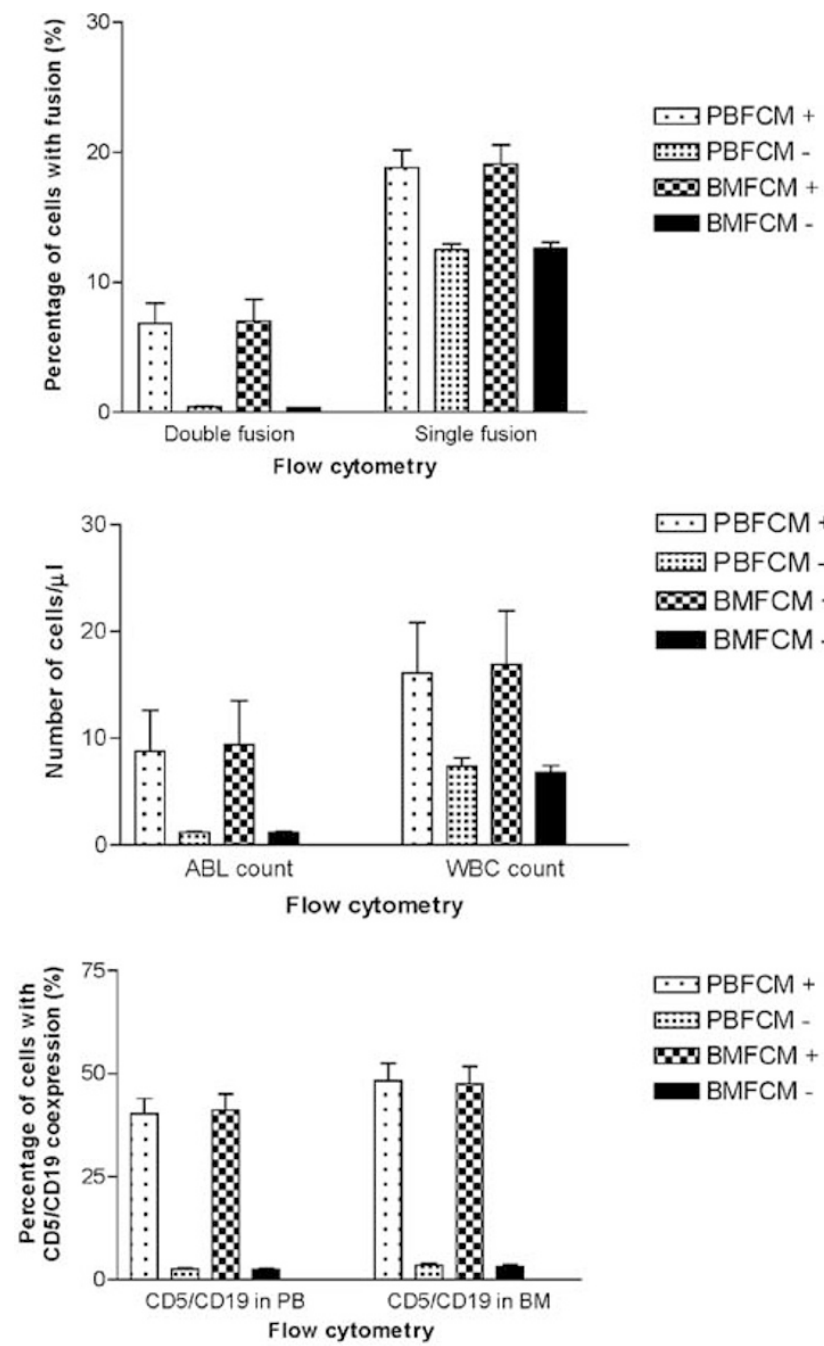

$\cdots$ PBFCM + 표료 PBFCM $\infty \mathrm{BMFCM}+$ BMFCM -

Figure 2 Cyclin D1/IgH-fusion signal pattern, absolute blood lymphocyte count, white blood cell count, and CD5/CD19 coexpression stratified by flow cytometry results in blood and corresponding bone marrow samples from patients with mantle cell lymphoma $(N=185)$. negative groups, flow cytometry-positive groups (both in the peripheral blood and the corresponding bone marrow samples) demonstrated significantly higher mean numbers of double-fusion-positive cells (6.8\% vs $0.4 \%$ in peripheral blood samples; $7.0 \%$ vs $0.3 \%$ in bone marrow samples; $P<0.01$ ) and singlefusion-positive cells $(18.8 \%$ vs $12.5 \%$ in peripheral blood samples; $19.1 \%$ vs $12.6 \%$ in bone marrow samples; $P<0.01$ ). Flow cytometry-positive groups, when compared to flow cytometry-negative groups, also had significantly higher mean numbers of CD5/ CD19-positive cells in the peripheral blood and the corresponding bone marrow samples $(P<0.01)$. Furthermore, the mean absolute blood lymphocyte and white blood cell counts in the peripheral blood and the corresponding bone marrow samples were significantly higher in flow cytometry-positive groups than in flow cytometry-negative peripheral blood and bone marrow samples $(P<0.01)$.

The FISH analysis results according to the percentages of double-fusion- and single-fusionpositive cells stratified by before and after first chemotherapy are summarized in Table 1 . Of the 185 mantle cell lymphoma peripheral blood samples, 56 $(30.3 \%)$ were collected before first chemotherapy while 129 (69.7\%) were collected after. Prechemotherapy mantle cell lymphoma peripheral blood samples had a mean percentage of $4.3 \%$ of doublefusion-positive cells and up to a maximum percentage of $55.5 \%$. In comparison, postchemotherapy mantle cell lymphoma peripheral blood samples had a mean percentage of $0.9 \%$ of double-fusionpositive cells and up to a maximum percentage of $33.0 \%$. Similarly, prechemotherapy peripheral blood samples had significantly higher percentages of single-fusion-positive cells compared to postchemotherapy peripheral blood samples $(P<0.01)$.

Interphase FISH-positivity in the peripheral blood samples is described in Table 2. Using the cutoffs described above, the samples were divided into four groups: double-fusion-positive and -negative and single-fusion-positive and -negative. Double-fusionand single-fusion-positive groups were more likely to have positive flow cytometry or morphologic results in the peripheral blood and the corresponding bone marrow samples for mantle cell lymphoma than double-fusion- and single-fusion-negative groups $(P<0.01)$.

The numbers of CD5/CD19-positive cells in the peripheral blood and the corresponding bone marrow samples are shown in Table 3 . When compared with double-fusion- and single-fusion-negative groups, double-fusion- and single-fusion-positive groups had significantly elevated mean percentages of CD5/CD19-positive cells in both the peripheral blood samples and the corresponding bone marrow samples, with the highest mean percentage of CD5/ CD19-positive cells being in the corresponding bone marrow samples.

The absolute blood lymphocyte and white blood cell counts in the peripheral blood samples are 
Table 1 Double- and single-fusion-positive cells in nonpaired peripheral blood samples before and after chemotherapy ( $N=185)$

\begin{tabular}{|c|c|c|c|c|c|c|c|}
\hline \multirow[t]{2}{*}{ Group } & \multirow[t]{2}{*}{ Number of samples } & \multicolumn{6}{|c|}{ Percentage of positive cells } \\
\hline & & Mean & s.d. & Min & $\operatorname{Max}$ & $95 \% C I$ & $\mathrm{P}^{\mathrm{a}}$ \\
\hline \multicolumn{8}{|l|}{ Double fusion } \\
\hline Prechemotherapy MCL & 56 & 4.3 & 8.8 & 0.0 & 55.5 & $1.9-6.6$ & $<0.01$ \\
\hline Postchemotherapy MCL & 129 & 0.9 & 3.2 & 0.0 & 33.0 & $0.3-1.4$ & - \\
\hline \multicolumn{8}{|l|}{ Single fusion } \\
\hline Prechemotherapy MCL & 56 & 17.1 & 8.7 & 0.0 & 54.0 & $14.8-19.4$ & $<0.01$ \\
\hline Postchemotherapy MCL & 129 & 12.6 & 5.1 & 0.0 & 24.0 & $11.6-13.5$ & - \\
\hline
\end{tabular}

${ }^{\mathrm{a} C o m p a r e d ~ w i t h ~ p o s t c h e m o t h e r a p y ~ m a n t l e ~ c e l l ~ l y m p h o m a ~ g r o u p . ~}$

s.d., standard deviation; Min, minimum; Max, Maximum; CI, confidence interval; MCL, mantle cell lymphoma.

Table 2 Comparison of FISH signal patterns to flow cytometry and morphology in peripheral blood and bone marrow

\begin{tabular}{|c|c|c|c|}
\hline \multirow{2}{*}{$\begin{array}{l}\text { Signal pattern } \\
\text { Double fusion + }\end{array}$} & \multicolumn{2}{|c|}{ Flow cytometry or morphology } & \multirow{2}{*}{$\begin{array}{c}\mathrm{P}^{\mathrm{a}} \\
<0.01\end{array}$} \\
\hline & $\begin{array}{c}\text { PB FCM+ } \\
30\end{array}$ & $\begin{array}{c}\text { PB FCM- } \\
11\end{array}$ & \\
\hline Double fusion - & 14 & 115 & - \\
\hline Single fusion + & $\begin{array}{l}\text { PB FCM+ } \\
32\end{array}$ & $\begin{array}{c}\text { PB FCM- } \\
46\end{array}$ & $<0.01$ \\
\hline Single fusion - & 12 & 80 & - \\
\hline Double fusion $+{ }^{b}$ & $\begin{array}{l}\text { PB MOR.+ } \\
18\end{array}$ & $\begin{array}{c}\text { PB MOR- } \\
14\end{array}$ & $<0.01$ \\
\hline Double fusion - & 23 & 103 & - \\
\hline Single fusion $+{ }^{b}$ & $\begin{array}{c}\text { PB MOR. + } \\
29\end{array}$ & $\begin{array}{l}\text { PB MOR- } \\
45\end{array}$ & $<0.01$ \\
\hline Single fusion - & 12 & 72 & - \\
\hline Double fusion + & $\begin{array}{c}\text { BM FCM+ } \\
28\end{array}$ & $\begin{array}{c}\text { BM FCM- } \\
9\end{array}$ & $<0.01$ \\
\hline Double fusion - & 13 & 102 & - \\
\hline Single fusion + & $\begin{array}{c}\text { BM FCM+ } \\
30\end{array}$ & $\begin{array}{c}\text { BM FCM- } \\
42\end{array}$ & $<0.01$ \\
\hline Single fusion - & 11 & 69 & - \\
\hline Dorble & BM MOR. + & BM MOR.- & 01 \\
\hline $\begin{array}{l}\text { Double fusion }+{ }^{\circ} \\
\text { Double fusion }-\end{array}$ & $\begin{array}{l}28 \\
23\end{array}$ & & $<0.01$ \\
\hline Single fusion $+{ }^{b}$ & $\begin{array}{c}\text { BM MOR. }+ \\
35\end{array}$ & $\begin{array}{c}\text { BM MOR.- } \\
41\end{array}$ & $<0.01$ \\
\hline Single fusion - & 16 & 69 & - \\
\hline
\end{tabular}

${ }^{\mathrm{a} C o m p a r e d}$ with the corresponding fusion-negative group. Some of the samples did not have flow cytometric studies and/or bone marrow biopsies available for evaluation.

${ }^{b}$ Double fusion+: double fusion in peripheral blood is $>1.5 \%$; Single fusion+: Single fusion in peripheral blood is $>14 \%$.

MOR, morphologic evidence of mantle cell lymphoma; PB, peripheral blood; BM, bone marrow.

shown in Table 4. The double-fusion-positive group had higher mean absolute blood lymphocyte and white blood cell counts in the peripheral blood samples than the double-fusion-negative group did $(P<0.01)$. The single-fusion-positive group also had higher mean absolute blood lymphocyte and white blood cell counts than did the single-fusion-negative group $(P=0.04)$. Of the 17 peripheral blood samples with double fusion $>5 \%$, 10 did not demonstrate elevated absolute blood lymphocyte or white blood cell counts.

The sensitivities, specificities, positive predictive values, and negative predictive values of the doubleand single-fusion FISH tests for detecting mantle cell lymphoma, which used flow cytometry as the gold standard, are described in Figure 3. Using flow cytometry as the gold standard, the sensitivity of single fusion compared to that of double fusion in detecting mantle cell lymphoma in the peripheral blood was slightly higher. However, the specificity of single fusion compared to that of double fusion was much lower. In addition, the positive predictive value of the FISH test was highest if $>1.5 \%$ doublefusion-positive cells were present in the peripheral blood

Although single-fusion positivity was associated with a high false-positive rate, single-fusion-positive cells were dominant in some cases when compared with double-fusion-positive ones. Notably, one of the mantle cell lymphoma samples had $36 \%$ singlefusion-positive cells but no double-fusion-positive cells. In combining double and single fusion by looking at the percentages of cells with at least one fusion signal (either double- or single-fusion), we found that the maximum peripheral blood involvement was $74 \%$. One mantle cell lymphoma case had $55.5 \%$ double-fusion-positive and $18 \%$ singlefusion-positive cells, while another had $20 \%$ double-fusion-positive and $54 \%$ single-fusion-positive cells. Assuming a false-positive rate of $10 \%$ for single fusion and $1 \%$ for double fusion, the maximum peripheral blood involvement in mantle cell lymphoma cases was around $65 \%$.

\section{Discussion}

Mantle cell lymphoma cells are usually positive for the cyclin D1 protein by immunohistochemistry and have CD5/CD19 coexpression but lack CD10 and CD23. Thus, flow cytometry analysis in conjunction with morphologic and immunocytochemical 
Table 3 CD5/CD19 coexpression by flow cytometry in double- and single-fusion-positive peripheral blood and corresponding bone marrow samples $(N=185)$

\begin{tabular}{|c|c|c|c|c|c|}
\hline Signal pattern & Number of samples & Mean (\%) & s.d. & $95 \% C I$ & $\mathrm{P}^{\mathrm{a}}$ \\
\hline \multicolumn{6}{|c|}{ Flow cytometry positive for CD5/CD19 coexpression in peripheral blood } \\
\hline Double fusion $+{ }^{b}$ & 41 & 33.6 & 29.5 & $24.3-42.9$ & $<0.01$ \\
\hline Double fusion - & 127 & 5.6 & 10.7 & $3.7-7.4$ & - \\
\hline Single fusion + & 78 & 20.2 & 27.0 & $14.1-26.3$ & $<0.01$ \\
\hline Single fusion - & 90 & 5.6 & 9.8 & $3.6-7.7$ & - \\
\hline \multicolumn{6}{|c|}{ Flow cytometry positive for CD5/CD19 coexpression in bone marrow } \\
\hline Double fusion + & 37 & 40.9 & 32.3 & $30.1-51.6$ & $<0.01$ \\
\hline Double fusion - & 114 & 7.0 & 13.2 & $4.5-9.4$ & - \\
\hline Single fusion + & 72 & 25.3 & 31.0 & $18.0-32.5$ & $<0.01$ \\
\hline Single fusion - & 79 & 6.2 & 9.8 & $4.0-8.4$ & - \\
\hline
\end{tabular}

${ }^{\mathrm{a} C}$ Compared with the corresponding fusion-negative group.

${ }^{\mathrm{b}}$ Double and single fusion measured in peripheral blood: double fusion+, double fusion in peripheral blood is $>1.5 \%$; and single fusion+, single fusion in peripheral blood is $>14 \%$.

s.d. standard deviation; CI, confidence interval.

Table 4 Absolute blood lymphocyte count and white blood cell count in double- and single-fusion-positive cells in peripheral blood samples $(N=185)$

\begin{tabular}{|c|c|c|c|c|c|}
\hline Signal pattern & Number of samples & $\operatorname{Mean}\left(\times 10^{3} \mathrm{cells} / \mu \mathrm{l}\right)$ & s.d. & $95 \% C I$ & $\mathrm{P}^{\mathrm{a}}$ \\
\hline \multicolumn{6}{|c|}{ Absolute blood lymphocyte count } \\
\hline Double fusion $+^{\mathrm{b}}$ & 42 & 7.9 & 25.2 & $0.1-15.8$ & $<0.01$ \\
\hline Double fusion - & 142 & 1.5 & 2.7 & $1.1-2.0$ & - \\
\hline Single fusion + & 84 & 5.0 & 18.3 & $1.1-9.0$ & 0.04 \\
\hline Single fusion - & 100 & 1.3 & 1.0 & $1.1-1.5$ & - \\
\hline \multicolumn{6}{|c|}{ White blood cell count } \\
\hline Double fusion + & 42 & 16.4 & 31.7 & $6.5-26.1$ & $<0.01$ \\
\hline Double fusion - & 142 & 8.5 & 11.2 & $6.6-10.3$ & - \\
\hline Single fusion + & 84 & 13.4 & 24.4 & $8.1-18.6$ & 0.04 \\
\hline Single fusion - & 100 & 7.7 & 10.2 & $5.7-9.8$ & - \\
\hline
\end{tabular}

${ }^{\mathrm{a} C o m p a r e d ~ w i t h ~ t h e ~ c o r r e s p o n d i n g ~ f u s i o n-n e g a t i v e ~ g r o u p . ~}$

$\mathrm{b}$ Double and single fusion measured in peripheral blood, double fusion+: double fusion in peripheral blood is $>1.5 \%$; and single fusion+: single fusion in peripheral blood is $>14 \%$.

s.d., standard deviation; CI, confidence interval.

analyses is used to diagnose most mantle cell lymphoma cases in tissue. We selected flow cytometry analysis as the gold standard for evaluating peripheral blood involvement of mantle cell lymphoma since some of the mantle cell lymphoma cases might be morphologically atypical or negative. The evaluation of peripheral blood involvement is complicated and may be affected by many factors. We focused on the evaluation of individual peripheral blood samples regardless of disease progression. Chemotherapy may decrease the percentage of lymphoma cells to undetectable levels in peripheral blood even while corresponding bone marrow samples are positive. Negative morphology in peripheral blood may be due to dilution factors or difficulty in distinguishing between normal lymphocytes and typical variants of mantle cell lymphoma. It may be easier to detect rare abnormal cells tagged with fusion signals using FISH probes than to detect a few malignant cells on a Romanowskystained peripheral blood smear. Furthermore, FISH may be very helpful when the morphology is not typical or when the flow cytometry results are suboptimal. Lymphocytosis may be an obvious indicator of the leukemic phase of disease, but it may also be nonspecific for the presence of mantle cell lymphoma, as a few cases without lymphocytosis in this series were both positive for fusion FISH and positive by bone marrow morphology.

Another major purpose of this study was to determine whether the number of cyclin D1/IgH fusion-positive cells in peripheral blood could be used to evaluate the effectiveness of treatment. The significant drop in the number of double-fusionpositive cells after first chemotherapy suggested that the therapy cleared circulating malignant cells from the peripheral blood in most cases. Although leukemic mantle cell lymphoma is associated with a worse clinical outcome, the correlation between occult leukemic mantle cell lymphoma and its clinical significance is still not clear since current molecular-cytogenetic method development outpaces clinical study in minimal residual disease. We hypothesize that if the number of fusion-positive 

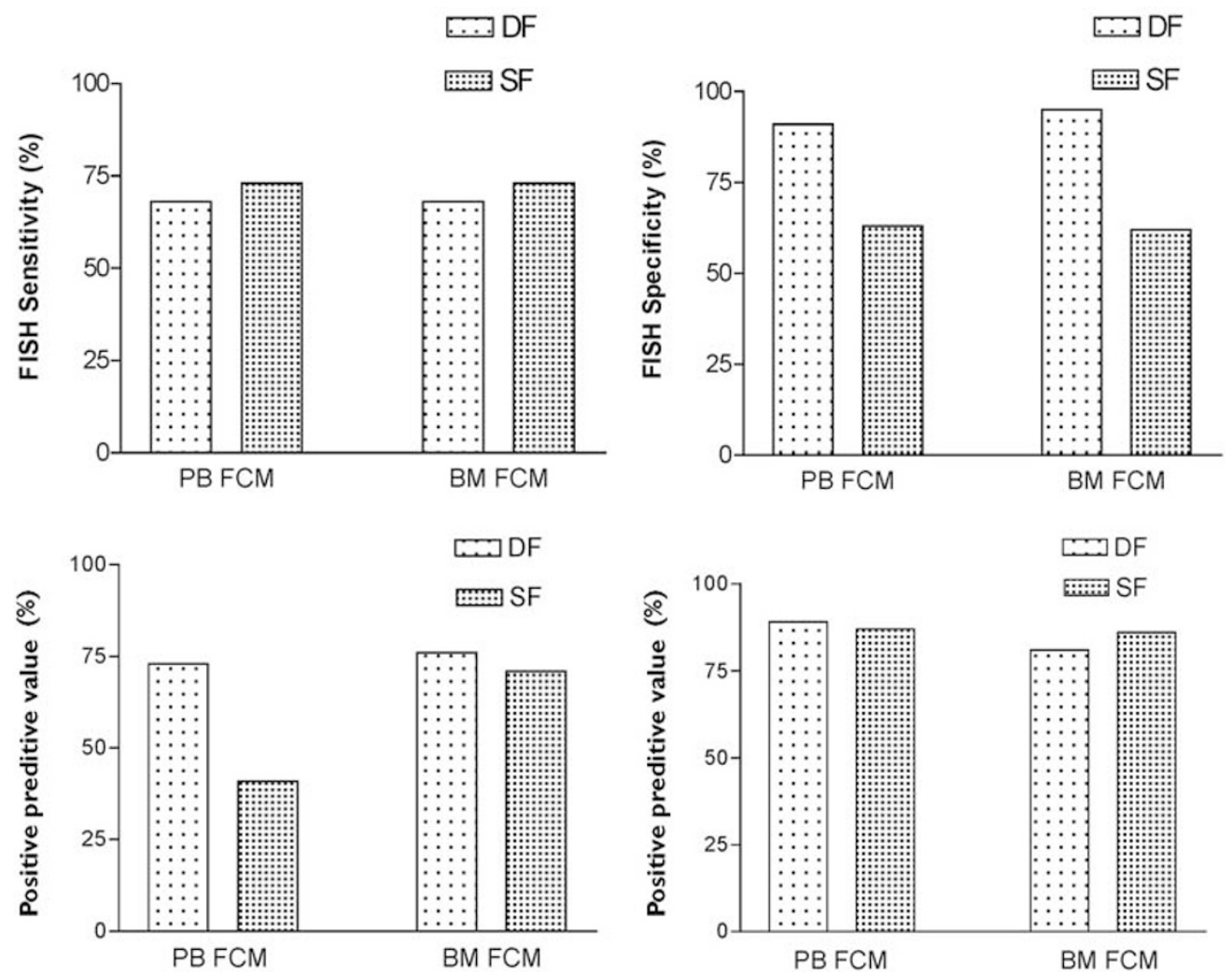

Figure 3 Sensitivity, specificity, positive predictive value, and negative predictive value of single and double fusion in peripheral blood by using flow cytometry results as gold standard $(N=185)$.

DF, double-fusion; SF, single-fusion; BM, bone marrow; FCM, flow cytometry; MOR, morphology.

cells increases over long-term follow-up, then recurrence will be likely. However, the present study looked only at the short-term effects of treatment and was not designed to predict recurrent disease. Few of the patients had evidence of recurrence at the time we collected the last peripheral blood sample. It will be interesting to follow these patients longer to evaluate the correlation between fusion FISH results and relapse.

Our results have shown the high specificity but not the high sensitivity of the double-fusion FISH test in peripheral blood. Conversely, the sensitivity of the single-fusion FISH test was slightly higher with lower specificity. The lower than expected sensitivities for both single and double fusion might be explained by the fact that peripheral blood is not always involved by lymphoma and we were using flow cytometry results as the gold standard in this study. However, some investigators have reported more than $90 \%$ sensitivity and specificity for FISH testing when they directly dealt with involved tissue such as fine-needle aspiration or biopsy samples from lymph nodes and used the initial clinical diagnosis as the gold standard. Negative predictive values were very consistent for either the single- or the double-fusion FISH test. The design of the probe allowed us to visualize the cyclin D1/IgH doublefusion that is an indication of reciprocal cyclin D1/IgH translocation. Double-fusion quantitation greatly reduces the false-positive rates of interphase FISH, thereby increasing the specificity of the procedure. Nevertheless, we observed a lower than expected sensitivity of the double-fusion FISH test when we used flow cytometry analysis as the gold standard. This is because of the fact that some mantle cell lymphoma cells may have cyclin D1/IgH translocations that are not reciprocal and seen as a single-fusion but still express cyclin D1, CD5, and CD19. Another possible reason could be that some rearranged segments covered by the fluorescent probe are too small to be visualized under the microscope, resulting in a single-fusion signal. Moreover, unlike the International System for Human Cytogenetic Nomenclature, currently no standardized interphase FISH signal quantitation system is available. Different investigators are using different criteria for signal quantitation. Some methods of quantitation are better than others in terms of FISH sensitivity.

We believe that some of the single-fusion-positive cells may be false-negative double-fusion cells, thus representing a subgroup of malignant cells with break points distal and/or proximal to the probe coverage area or a cryptic translocation. To overcome this disadvantage, the counting of only singlefusion cells with either extra cyclin D1 or IgH signals (a fusion plus extra dots approach) as positive cells could be used in order to improve 
the sensitivity of the test since extra signals may represent rearrangement of either cyclin $D 1$ or $I g H$ gene loci and are more likely to be true positive cells. A low percentage of single-fusion-positive cells without any double fusion-positive cells is equivocal. The significance of marginally elevated percentages of cells with single-fusion but not double fusion signals in the absence of positive mantle cell lymphoma morphology is uncertain. These single-fusion-positive cells may represent minimal residual disease or a precursor to minimal residual disease; however, follow-up studies will be necessary to confirm this hypothesis.

Our study demonstrated that the results of the well-established FISH method correlated well with those of the traditional gold standard flow cytometry for mantle cell lymphoma, therefore validating its application in detecting mantle cell lymphoma involvement in peripheral blood. We found that using FISH on peripheral blood samples was capable of detecting low numbers of circulating fusion-positive malignant cells and, therefore, could complement currently available tests to detect mantle cell lymphoma. Also, obtaining peripheral blood instead of bone marrow samples is far easier and less painful than obtaining bone marrow. Therefore, the collection and testing by FISH of peripheral blood for mantle cell lymphoma may offer a reliable and convenient way to accurately and quickly assess minimal involvement of mantle cell lymphoma in peripheral blood.

\section{Acknowledgements}

We thank Katie Matias for editing this manuscript and Dr John Proffitt for his technical input. This study is supported by the Hickman/Cook Fund and Hudson ARD Memorial Fund, 2002.

\section{References}

1 Zukerberg LR, Medeiros LJ, Ferry JA, et al. Diffuse lowgrade B-cell lymphomas. Four clinically distinct subtypes defined by a combination of morphologic and immunophenotypic features. Am J Clin Pathol 1993;100:373-385.

2 Caligaris-Cappio F, Gobbi M, Bofill M, et al. Infrequent normal B lymphocytes express features of B-chronic lymphocytic leukemia. J Exp Med 1982;155:623-628.

3 Abe M, Tominaga K, Wakasa H. Phenotypic characterization of human B-lymphocyte subpopulations, particularly human CD5+ B-lymphocyte subpopulation within the mantle zones of secondary follicles. Leukemia 1994;8:1039-1044.

4 Dorfman DM, Pinkus GS. Distinction between small lymphocytic and mantle cell lymphoma by immunoreactivity for CD23. Mod Pathol 1994;7:326-331.

5 Kilo MN, Dorfman DM. The utility of flow cytometric immunophenotypic analysis in the distinction of small lymphocytic lymphoma/chronic lymphocytic leuke- mia from mantle cell lymphoma. Am J Clin Pathol 1996;105:451-457.

6 Kumar S, Green GA, Teruya-Feldstein J, et al. Use of CD23 (BU38) on paraffin sections in the diagnosis of small lymphocyte lymphoma and mantle cell lymphoma. Mod Pathol 1996;9:925-929.

7 Dunphy CH, Wheaton SE, Perkins SL. CD23 expression in transformed small lymphocytic lymphomas/chronic lymphocytic leukemias and blastic transformations of mantle cell lymphoma. Mod Pathol 1997;10:818-822.

8 Medeiros LJ, Van Krieken JH, Jaffe ES, et al. Association of bcl-1 rearrangements with lymphocytic lymphoma of intermediate differentiation. Blood 1990;76:2086-2090.

9 Vandenberghe E, De Wolf-Peeters C, van den Oord J, et al. Translocation $(11 ; 14)$ : a cytogenetic anomaly associated with B-cell lymphomas of non-follicle centre cell lineage. J Pathol 1991;163:13-18.

10 Williams ME, Westermann CD, Swerdlow SH. Genotypic characterization of centrocytic lymphoma: frequent rearrangement of the chromosome 11 bcl-1 locus. Blood 1990;76:1387-1391.

11 Yang WI, Zukerberg LR, Motokura T, et al. Cyclin D1(Bcl-1, PRAD1) protein expression in low-grade Bcell lymphomas and reactive hyperplasia. Am J Pathol 1994;145:86-96.

12 Pittaluga S, Verhoef G, Criel A, et al. Prognostic significance of bone marrow trephine and peripheral blood smears in 55 patients with mantle cell lymphoma. Leuk Lymphoma 1996;21:115-125.

13 Singleton TP, Anderson MM, Ross CW, et al. Leukemic phase of mantle cell lymphoma, blastoid variant. Am J Clin Pathol 1999;111:495-500.

14 Viswanatha DS, Foucar K, Berry BR, et al. Blastic mantle cell leukemia: an unusual presentation of blastic mantle cell lymphoma. Mod Pathol 2000;13: 825-833.

15 Wong KF, Chan JK, So JC, et al. Mantle cell lymphoma in leukemic phase: characterization of its broad cytologic spectrum with emphasis on the importance of distinction from other chronic lymphoproliferative disorders. Cancer 1999;86:850-857.

16 Bosch F, Lopez-Guillermo A, Campo E, et al. Mantle cell lymphoma: presenting features, response to therapy, and prognostic factors. Cancer 1998;82: 567-575.

17 Coiffier B. Which treatment for mantle-cell lymphoma patients in 1998? J Clin Oncol 1998;16:3-5.

18 Teodorovic I, Pittaluga S, Kluin-Nelemans JC, et al. Efficacy of four different regimens in 64 mantle-cell lymphoma cases: clinicopathologic comparison with 498 other non-Hodgkin's lymphoma subtypes. European Organization for the Research and Treatment of Cancer Lymphoma Cooperative Group. J Clin Oncol 1995;13:2819-2826.

$19 \mathrm{Li} \mathrm{JY,} \mathrm{Gaillard} \mathrm{F,} \mathrm{Moreau} \mathrm{A,} \mathrm{et} \mathrm{al.} \mathrm{Detection} \mathrm{of}$ translocation $\mathrm{t}(11 ; 14)(\mathrm{q} 13 ; \mathrm{q} 32)$ in mantle cell lymphoma by fluorescence in situ hybridization. Am J Pathol 1999;154:1449-1452.

20 Remstein ED, Kurtin PJ, Buno I, et al. Diagnostic utility of fluorescence in situ hybridization in mantle cell lymphoma. Br J Haematol 2000;110:856-862.

21 Frater JL, Tsiftsakis EK, Hsi ED, et al. Use of novel $t(11 ; 14)$ and $t(14 ; 18)$ dual-fusion fluorescence in situ hybridization probes in the differential diagnosis of lymphomas of small lymphocytes. Diagn Mol Pathol 2001;10:214-222. 